\title{
Enhancement of Extender Excellence of Frozen Bull Semen Using a-Tocopherol as an Antioxidant
}

\author{
Zakir Ullah', Hamayun Khan', Syeda Maryam Hussain², Muhammad Tarique Tunio³, \\ Syed Muhmmad Raihan Dilshad ${ }^{4}$, Ali Gohar ${ }^{5}$, Hafsa Zahid5 \& Abid Ali ${ }^{5}$
}

\begin{abstract}
Background: Addition of the antioxidant to extender media is the most appropriate attempt to reduce structural losses encounter during the process of cryopreservation. Hence semen excellence could be maintained for longer duration without adverse impact. Additionally antioxidants are not only capturing the reactive oxygen species but also improve the sperm quality indicators and fertility. Accordingly, current elucidation has been executed to explore the dose depended appraisal of varied concentration of $\alpha$-tocopherol in Tris-based extender on frozen-thawed bull semen quality parameters for enhancement of bull semen cryopreservation in the subtropical ecosystem of Peshawar.

Materials, Methods \& Results: Experiments were carried out on semen that has been collected from both Achai-an indigenous breed and Holstein Friesian (HF) - the exotic breed in artificial vagina maintained at $42^{\circ} \mathrm{C}$ from the experimental bulls of either breed and processed independently breed wise. Semen specimens with above $70 \%$ motility were evaluated separately breed-wise under same environmental condition. Standard procedure was adopted to extend the collected semen in the experimental extenders and frozen subsequently. After thawing, further Analysis of the frozen straws of semen was carried out for sperm excellence indicators that include motility, viability, acrosomal integrity and functional integrity of spermatozoa under the subtropical condition. Sperm viability and acrosomal integrity were determined by dual staining procedure i.e. Trypan-blue and Giemsa stains. The hypo-osmotic swelling (HOS) test was used to assess plasma membrane integrity. The current elucidation demonstrated that $\alpha$-tocopherol $1.5 \mathrm{mg} / \mathrm{mL}$ supplemented in extender had significantly $(P<0.05)$ increased sperm excellence gauge that includes motility, viability, acrosomal integrity and functional membrane integrity in both the breeds. On the other hand, the result further elucidated those concentrations higher or lower than $1.5^{-1}$ $\mathrm{mg} / \mathrm{mL} \alpha$-tocopherol supplemented in the present study resulted in a lower semen functional attributes subsequent to freezing. Discussion: Biological samples were preserved by various methods such cryopreservation at low temperature to maintain them intact and facilitate downstream experiments. Antioxidants were associated with the chemical breakage of the substrate consequential from oxidation and counterbalance the free radicals thus diminish the damaging impacts to spermatozoa in cryopreservation. There is lack of data with respect to lipid peroxidation and cell reinforcement limit in cryopreserved semen, and cryopreservation is related with the generation of receptive oxygen species (ROS) which lead to lipid peroxidation (LPO) of sperm membranes, bringing about lost motility, viability and fertility of sperm. Recently, in the reproductive management of the dairy animals, an important focus of research is to overcome the deleterious impact associated with semen cryopreservation. Elucidation of differential dose depended expression of $\alpha$-tocopherol in semen extender has been evident in this study. The results were corroborated with diverse mammalian species under varied ecological management setups. Furthermore the detrimental impact of high doses of $\alpha$-tocopherol in the semen extender has been demonstrated. It was deduced from the current finding that addition of $\alpha$-tocopherol via dose depended approach in Tri extender enhanced the cryosurvivability of both Achai indigenous breed and HF the exotic breed, under existing reproductive management in the local ecosystem of Peshawar.
\end{abstract}

Keywords: Achai, Holstein Friesian, motility, $\alpha$ - tocopherol.

DOI: $10.22456 / 1679-9216.90447$ ${ }^{3}$ Department of Agricultural Sciences, Allama Iqbal Open University, Islamabad, Pakistan. ${ }^{4}$ Department of Clinical Sciences, Faculty of Veterinary and Animal Sciences, Gomal University, Dera Ismail Khan, Pakistan. ${ }^{5}$ College of Veterinary Science and Animal Husbandry \& Department of Zoology, Abdul Wali Khan University Mardan, Khyber Pakhtunkhwa, Pakistan. CORRESPONDENCE: A. Ali [uop_ali@yahoo.com - Fax: +92 (937) 843357]. Department of Zoology. Abdul Wali Khan University Mardan. ZC 23200 Khyber Pakhtunkhwa, Pakistan. 


\section{INTRODUCTION}

Affluent fertility is one of the most imperative and preferred segments of a modern cattle breeding program in the reproductive management of dairy enterprise. Achai cattle are the smallest among the local cattle breed in Pakistan that has been developing from the Himalayan Hindukush hilly area [24]. The distinctiveness of this breed is its capability to flourish well in the mountainous ecosystem in conjunction with ruthless topographical grazing in comparison with exotic breeds of cattle from the temperate area. Additionally, Achai cattle are being reared by the mountainous agro-pastoral communities in large number and could make available space for their sustainable conservation. Also, semen cryopreservation has been considered an important tool for the preservation of genetic assets [25]. To this end, supplementation of $\alpha$-tocopherol in semen extender biology against ROS and damages in semen freezing has been recently reported in diverse mammalian species in the varied ecosystem of the world [16,3].

To date, no defined documents are available on the use of $\alpha$-tocopherol as an antioxidant in frozen bull semen preservation in the subtropical environment of Peshawar. Accordingly, the current endeavour has been elucidated to establish the most positive level of $\alpha$-tocopherol for cryopreservation of Achai and Holstein Friesian bull semen, breed-wise separately under the subtropical condition of Peshawar region to elaborate shielding impact of $\alpha$-tocopherol on the structural protection of bull semen against the unfavourable environment of cryopreservation for optimization of the fertility management and the efficacy of breeding programs in local cattle breeds.

\section{MATERIALS AND METHODS}

\section{Samples}

Experiments were carried out on semen of six matured bulls, three Achai - the indigenous breed and three Holstein Friesian - exotic breed at Government Cattle Breeding and Dairy Farm Harichand Charsadda, Khyber Pakhtunkhwa, Pakistan.

\section{Methodology}

Artificial vagina maintained at $42^{\circ} \mathrm{C}$ was used for collection of Semen from the experimental bulls of either breed and processed independently breed wise. Two successive ejaculates were collected from each bull at weekly interval for 3 weeks in April- May. Gross and microscopic examination of the ejaculates was appraised soon after collection. Semen specimens with above $70 \%$ motility were chosen for advance analysis. The semen was split into four aliquots in which triscitric acid extender (TCA) supplemented with various concentrations of $\alpha$-tocopherol was used to reach $0 \mathrm{mg} /$ $\mathrm{mL}$ (group I, control), $0.5 \mathrm{mg} / \mathrm{mL}$ (group II), $1.0 \mathrm{mg} /$ $\mathrm{mL}$ (group III), $1.5 \mathrm{mg} / \mathrm{mL}$ (group IV), $2.0 \mathrm{mg} / \mathrm{mL}$ (group V), $2.50 \mathrm{mg} / \mathrm{mL}$ (group VI) and $3.0 \mathrm{mg} / \mathrm{mL}$ (group VII) [Table 1]. Diluted semen was cooled to $4^{\circ} \mathrm{C}$ for $2 \mathrm{~h}$, equilibrated for $4 \mathrm{~h}$ at $4^{\circ} \mathrm{C}$, filled in straws $(0.5$ $\mathrm{mL}$ ) at $4^{\circ} \mathrm{C}$, kept in liquid nitrogen vapours for $10 \mathrm{~min}$ and subsequently held in the liquid nitrogen $\left(-196^{\circ} \mathrm{C}\right)$ for assessment. Post-thawed motility of frozen semen straws was assessed using the standard procedure. The hot-water bath was used for $30 \mathrm{~s}$ for Thawing of frozen semen straws at $37^{\circ} \mathrm{C}$.

Table 1. Concentration of freezing extender with various concentration of $\alpha$-tocopherol.

\begin{tabular}{cc}
\hline Group & Antioxidant (per mL of extender) \\
\hline Group-I & Control $=0.0$ \\
Group-II & $\alpha$-tocopherol $=0.50 \mathrm{mg} / \mathrm{mL}$ \\
Group-III & $\alpha$-tocopherol $=1.0 \mathrm{mg} / \mathrm{mL}$ \\
Group-IV & $\alpha$-tocopherol $=1.5 \mathrm{mg} / \mathrm{mL}$ \\
Group-V & $\alpha$-tocopherol $=2.0 \mathrm{mg} / \mathrm{mL}$ \\
Group-VI & $\alpha$-tocopherol $=2.5 \mathrm{mg} / \mathrm{mL}$ \\
Group-VII & $\alpha$-tocopherol $=3.0 \mathrm{mg} / \mathrm{mL}$ \\
\hline
\end{tabular}

Sperm viability and acrosomal integrity were determined by dual staining procedure i.e. Trypanblue and Giemsa stains. Live and dead spermatozoa were differentiated via the supravital stain trypan-blue whereas Giemsa integrity of the acrosomal membrane was assessed through Giemsa stain. Likewise, the hypo-osmotic swelling (HOS) test was used to assess plasma membrane integrity.

\section{Statistical analysis}

The data were statistically analyzed with twoway analysis of variance using SPSS (Version 16) ${ }^{1}$. Duncan's Multiple Range Test was applied among the treated groups to make clear the level of significance.

\section{RESULTS}

During the current study, the progressive motility and viability of spermatozoa were significantly higher in $1.5 \mathrm{mg}$ or $1.0 \mathrm{mg} \alpha$-tocopherol supplemented 
groups in comparison with $0.5 \mathrm{~mm}$ or $0.00 \mathrm{mM}$ in both Achai and Holstein Friesian. Also, the current finding indicated that the number of HOST+ve spermatozoa at 1.5 or $1.0 \mathrm{mg}$ was greater when compared with the lower concentration of 0.5 or $0.00 \mathrm{mg} \alpha$-tocopherol. Furthermore, the significant positive impact for acrosomal integrity was recorded in semen samples treated with $1.5 \mathrm{mg} \alpha$-tocopherol followed by $1 \mathrm{mg}$, $0.5 \mathrm{mg}$ when the comparison was made with other treated concentration. On the other hand, the result were further elucidated that concentrations higher or lower than 1.5-1 mg/mL $\alpha$-tocopherol supplemented in the present study resulted in a lower semen functional attributes subsequent to freezing (Figure 1). The current study demonstrated that $1.5 \mathrm{mg} / \mathrm{mL}$ were the most favourable concentration of $\alpha$-tocopherol to be added to the TCA extender for improving the quality of frozen-thawed Achai and Holstein Frisian bull semen under the subtropical ecosystem of the Peshawar region (Table 1).

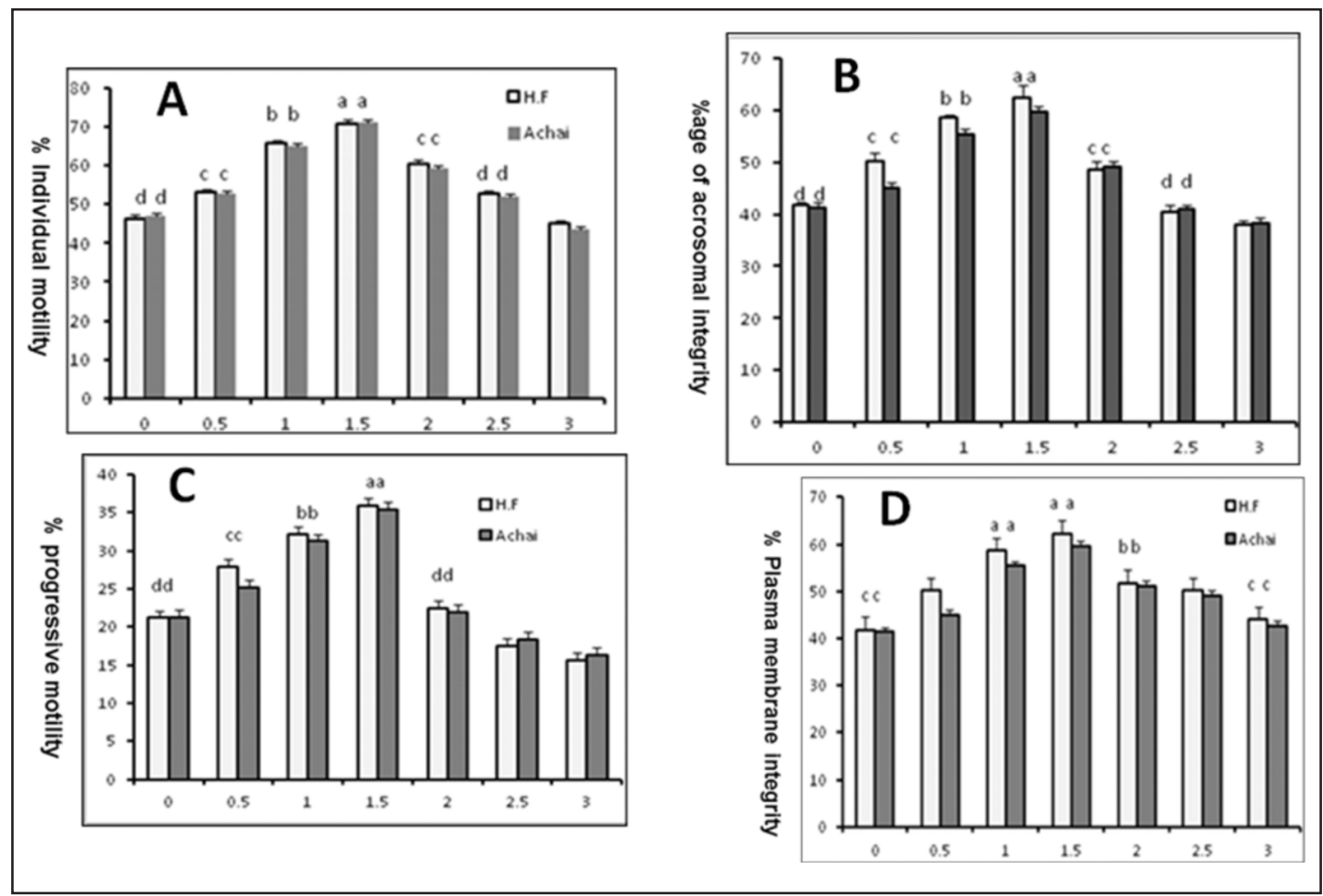

Figure 1. A,B,C and D indicated Post thaw functional assays of different level of $\alpha$-tocopherol in semen egg yolk extender. Bar indicated stander error, a,b,c and d indicted significant level at $0.05 \%$ among varied treated groups for fertility marker (motility and plasma membrane integrity) of post-thaw spermatozoa of $\mathrm{HF}$ and achai bull at diverse deliberation of $\alpha$-tocopherol In semen extender.

\section{DISCUSSION}

The current elucidation was executed to appraise which concentration of $\alpha$-tocopherol would provide the most effective protection against cold shock during the cryopreservation of bull spermatozoa in the subtropical environment of the Peshawar. The $\alpha$-tocopherol supplementation in the extender resulted in positive effects on bovine sperm motility, acrosome integrity and plasma membrane integrity, after thawing in this study. The beneficial effects of $\alpha$-tocopherol can be attributed to the antioxidant characteristics. $\alpha$-tocopherol supplementation in the extender reduced the lipid peroxidation potential and improved semen quality during the freezing-thawing [8]. Since an enormous amount of unsaturated fatty acids are abundantly available in spermatozoa cell membrane which is always a threat to the lipid peroxidation. The higher rate of lipid peroxidation leads to membrane damages, disorders of cell functions and decrease sperm motility [6]. In the current study, the supplementation of $\alpha$-tocopherol concentration rates of $1.50 \mathrm{mg}$ and $1 \mathrm{mg} / \mathrm{mL}$ significantly increased percent sperm motility in both the breed. These findings were similar to reported data in diverse mammalian species i.e. in Murrah buffalo bulls [23] and bovine [13]. Thus it seems reasonable from these studies in conjunction 
with the current finding that enhanced sperm motility by $\alpha$-tocopherol might be attributed to the antioxidant accomplishment of $\alpha$-tocopherol that scavenges the too much ROS production in lipid peroxidation [5].

This study further demonstrate a significant enhancement in sperm viability by addition of 1.5-1 $\mathrm{mg} / \mathrm{mL} \alpha$-tocopherol in freezing extenders. These results were corroborated with findings that have been documented in recent past in water buffaloes, ram and boar $[5,9,22]$. Taken these reports together with current finding, it seems rational that the potential reason behind the improved viability via $\alpha$-tocopherol supplementation in the semen extender biology might be antioxidant capacity of this antioxidant to minimize the concentration of lipid peroxidation by interrelating within chain in the course of action of oxidative stress [7,15,17].

Appraisal of the Acrosomal integrity of spermatozoa is among one of most important gauge for the assessment of the serviceable grade of the membrane [27]. In the recent past, it has been documented that hasty cryocapacitation of spermatozoa with typical cryopreservation ahead of freezing is associated with alteration in the acrosomal integrity, resulting in the inability of the spermatozoa for fertilization of eggs [22]. Correspondingly, supplementation of $\alpha$-tocopherol with a dose of 1.5 and $1 \mathrm{mg} / \mathrm{mL}$ in the current study has appreciably enhanced sperm acrosomal integrity in the semen extender for both Achai and Holstein Friesian. The obtained results in the current study were in accordance with the preceding findings in diverse mammalian species i.e. domestic pigs, bulls and boars $[4,19,22,28]$. Thus taken together these elucidation in conjunction with obtained result in the current study, the most probable rational of advanced acrosome integrity rate might be due to the antioxidant characteristics of $\alpha$-tocopherol that has been associated with maintenance of spermatozoa acrosomal integrity via diminution of the too much ROS formed throughout the cryopreservation procedure [20,21].

It is well established that the plasma membrane of spermatozoa is one of the essential components for successful mammalian fertilization [14]. Generally, the plasma membrane of spermatozoa is vulnerable to structural damages during the cold shock, the process wherein commencement of the succession response of free radical occurs [2,10]. Likewise, $\alpha$ - tocopherol supplementation with a dose of 1.5 or $1 \mathrm{mg} / \mathrm{mL}$ in Tris-based extender has improved post-thaw sperm plasma membrane integrity. The present findings were corroborated with findings that have been reported in the recent past in diverse mammalian species i.e., in bucks, bovine bulls and boars [13,16,22,29]. Thus, it seems reasonable from theses researches including the recent data, that $\alpha$-tocopherol might be associated with enhancement of the plasma membrane integrity in opposition to surfeit ROS formation as an outcome of cryopreservation and thawing of spermatozoa [7].

On the other hand, interestingly in the present study, Concentrations higher or lower than 1.5-1 mg/ $\mathrm{mL} \alpha$-tocopherol supplemented in the present study resulted in a lower semen quality subsequent to freezing. This indicates that $1-1.5 \mathrm{mg} / \mathrm{mL}$ as the recommended concentration of $\alpha$-tocopherol for optimum cryopreservation results in the subtropical environment. The rising deliberation from 0 to $1.5 \mathrm{mg} / \mathrm{mL}$ of $\alpha$-tocopherol in semen extender demonstrated progressive tendency in post-thaw spermatozoa excellence characteristics. It is indicative that these concentrations might be assisted in the enhancement of plasma membrane fluidity and integrity that are associated with a reduction of ice crystal manufacture in freezing process [20]. On contrary, the higher doses of $\alpha$-tocopherol have been associated with the deleterious impact on sperm survivability and functionality during the freezing-thawing process and/ or cryopreservation. In diverse mammalian species, the detrimental impact of high doses of $\alpha$ - tocopherol in the semen extender has been documented including the report of in rams and in water buffalo bulls $[9,26]$. The exact functional mechanism of a high dose of $\alpha$-tocopherol in the semen extender biology with detrimental impact is not yet clarified. However, one of the potential cause of deleterious impact on sperm might be due to toxic dose intensity that has been associated with the demolition of diverse procedure e.g. cellular respiration in mitochondria in mammalian spermatozoa [12]. The other potential reasons for the higher concentrations than the recommended deliberation of $1-1.5 \mathrm{mg} / \mathrm{mL}$ in the semen extender did not exert better impact on sperm functional characteristics might be attributed to the abundant availability of fatty acids that make mammalian sperm susceptible to lipid peroxidation $[1,18]$. Elucidation with a more high concentration of the $\alpha$-tocopherol in different semen extender under diverse environmental ecosystem would certainly clarify the functional mechanism for the deleterious impact in varied mammalian species. 


\section{CONCLUSION}

This study demonstrated that adding $\alpha$-tocopherol in tris based extender enhanced the post-thawed quality of semen. A concentration of 1.5 $\mathrm{mg} / \mathrm{mL}$ of $\alpha$-tocopherol was found to be the optimum level for best semen cryopreservation result using Tri semen extender. Additionally current study indicated that $\alpha$-tocopherol exerted its effects in a dose-dependent approach and at high concentration of $2.0 \mathrm{mg} /$ $\mathrm{mL}$ or more is drastic for post thaw sperm functional characteristics. On the other hand, the artificial insemination (AI) has paramount functional relevance in the animal reproduction for appraisal of whether the spermatozoa maintain the fertility capabilities or not. Further studies would be essential to corroborate the outcome of $\alpha$-tocopherol on the field fertility of cryopreserved Achai and Holstein Frisian sperm in existing management ecosystem and to resolve the potential reasons for the deleterious impact of the high doses $\alpha$-tocopherol on post-thaw functional indicators in semen extender biology taken into account the current finding along with earlier studies in varied ecosystem of the world.

\section{MANUFACTURER}

${ }^{1}$ Statistical Package for the Social Sciences (SPSS). Chicago, IL, USA.

Acknowledgements. We acknowledge the financial support of Pakistan Science Foundation for research facilities.

Declaration of interest. The authors declare no commercial or financial relationships that could be construed as a potential conflict of interest.

\section{REFERENCES}

1 Aitken R.J., Harkiss D. \& Buckingham D. 1993. Relationship between iron-catalyzed lipid-peroxidation potential and human sperm function. Journal of Reproduction Fertility. 98: 257-265.

2 Alkhedaide A., Alshehri Z.S., Sabry A., Abdel-Ghaffar T., Soliman M.M. \& Attia H. 2016. Protective effect of grape seed extract against cadmium-induced testicular dysfunction. Molecular Medical Reproduction. 13: 3101-3109.

3 Amini Pour H., Tahmasbi A.M. \& Naserian A.A. 2013. The influence of vitamin E on semen characteristics of ghezel rams in during cooling and frozen process. European Journal of Zoological Research. 2: 94-99.

4 Andrabi S.M.H., Ansari M.S., Ullah N. \& Afzal M. 2008. Effect of non-enzymatic antioxidants in extender on postthaw quality of buffalo (Bubalus bubalis) bull spermatozoa. Pakistan Veterinary Journal. 28: 159-162.

5 Anghel A.H., S. Zamfirescu., Coprean D. \& D. Nadolu. 2010. Antioxidant additives effect on cytological parameters of refrigerated ram semen. Lucrari. Science Journal. 53: 294-298.

6 Ball B.A., Medina V., Gravance C.G. \& Baumber J. 2001. Effect of antioxidants on preservation of motility, viability and acrosomal integrity of equine spermatozoa during storage at $5^{\circ} \mathrm{C}$. Theriogenology. 56: 577-589.

7 Beconi M.T., Francia C.R., Mora N.G. \& Affranchino M.A. 1993. Effect of natural antioxidants on frozen bovine semen preservation. Theriogenology. 40: 841-851.

8 Bucak M.N., Ateşşahin A., Varişli O., Yüce A., Tekin N. \& Akçay A. 2007. The influence of trehalose, taurine, cysteamine and hyaluronan on ram semen. Microscopic and oxidative stress parameters after freeze-thawing process. Theriogenology. 67: 1060-1067.

9 Dorostkar K., Alavi-Shoushtari S.M. \& Mokarizadeh A. 2012. Effects of in vitro selenium addition to the semen extender on the spermatozoa characteristics before and after freezing in water buffaloes (Bubalus bubalis). Veterinary Research Forum. 3: 263-268.

10 Fonseca J.F., Torres C.A.A., Maffili V.V., Borges A.M., Santos A.D.F., Rodrigues M.T. \& Oliveira R.F.M. 2005. The hypo-osmotic swelling test in fresh goat spermatozoa. Journal of Animal Reproduction. 2: 139-144.

11 Hashem N.M., El-Hady A.A. \& Hassan O. 2013. Effect of vitamin E or propolis supplementation on semen quality, oxidative status and hemato-biochemical changes of rabbit bucks during the hot season. Livestock Science. 157: 520-526.

12 Hawkes W.C. \& Turek P.J. 2001. Effects of dietary selenium on sperm motility in healthy men. Journal of Andrology. 22: 764-772.

13 Hu J.H., Zan L.S., Zhao X.L., Li Q.W., Jiang Z.L., Li Y.K. \& Li X. 2010. Effects of trehalose supplementation on semen quality and oxidative stress variables in frozen-thawed bovine semen. Journal of Animal Science. 88: 1657-1662.

14 Jeyendran R.S., van der Ven H.H., Perez-Pelaez M., Crabo B.G. \& Zaneveld L.J.B. 1984. Development of an assay to assess the functional integrity of the human sperm membrane and its relationship to other semen characters. Journal of Reproduction Fertility. 70: 219-228. 
15 Kadirvel G., Kumar S. \& Kumaresan A. 2009. Lipid peroxidation, mitochondrial membrane potential and DNA integrity of spermatozoa in relation to intracellular reactive oxygen species in liquid and frozen-thawed buffalo semen. Animal Reproduction Science. 114: 125-134.

16 Kamran D., Alavi-Shoushtari S.M. \& Mokarizadeh A. 2012. Effects of in vitro selenium addition to the semen extender on the spermatozoa characteristics before and after freezing in water buffaloes (Bubalus bubalis). Veterinary Research Forum. 4: 263-268.

17 Khan R.U., Rahman Z.U., Javed I. \& Muhammad F. 2012. Effect of vitamins, probiotics and protein on semen traits in post-molt male broiler breeders. Animal Reproduction Science. 135: 85-90.

18 Kothari S., Thompson A., Agarwal A. \& du Plessis S.S. 2010. Free radicals: Their beneficial and detrimental effects on sperm function. Indian Journal of Experimental Biology. 5: 425-435.

19 Moghbeli M., Kohram H., Zare-Shahaneh A., Zhandi M., Sharafi M., Nabi M.M., Zahedi V. \& Sharideh H. 2016. Are the optimum levels of the catalase and vitamin $\mathrm{E}$ in rooster semen extender after freezing-thawing influenced by sperm concentration? Cryobiology. 72: 264-268.

20 Nasiri A.H., Towhidi A. \& Zeinoaldini S. 2012. Combined effect of DHA and atocopherol supplementation during bull semen cryoprese rvation on sperm characteristics and fatty acid composition. Andrologia. 44: 550-555.

21 Ordonez I. 2008. Systemic abnormalities by a deficiency of Vitamin E. Thesis. School of Dentistry. Universidad Nacional Autonoma de Mexico. 14: 29-39.

22 Pech-Sansores A.G., Centurión-Castro F.G., Rodríguez-Buenfil J.C., Segura-Correa J.C. \& Aké-Lopez J.R. 2011. Effect of the addition of seminal plasma, vitamin $E$ and incubation time on post-thawed sperm viability in boar Semen. Tropical and Subtropica Agroecosystem. 14: 965-971.

23 Raina V.S., Gupta A.K. \& Singh K. 2002. Effect of antioxidant fortification on preservability of buffalo semen. AsianAustralian Journal of Animal Science. 15: 16- 18.

24 Saleem M., Rahim I., Rueff H., Khan M., Maselli D. \& Wiesmann U. 2010. Mountain cattle breed for coping with climate change: needs for conserving and reintroducing the Achai in the Hindu Kush Mountains of Northern Pakistan. Conference on International Research on Food Security, Natural Resource Management and Rural Development Tropentag. ETH Zurich. September 14-16. 4p.

25 Schäfer-Somi S., Kluger S., Knapp E., Klein D. \& Aurich C. 2006. Effects of semen extender and semen processing on motility and viability of frozen-thawed dog spermatozoa. Theriogenology. 66: 173-182.

26 Seremak B., Udala J. \& Lasota B. 1999. Influence of selenium additive on ram semen freezing quality. Electronic Journal of Polish Agricultural Universities. 2: 1-5.

27 Silva P.F.N. \& Gadella B.M. 2006. Detection of damage in mammalian sperm cells. Theriogenology. 65: 958-978.

28 Uc N., Centurión F., Alfaro M., Rodríguez. J. \& Sarmiento L. 2010. The addition of atocopherol and ascorbic acid to the freezing media and their effects on viability of post thawed swine semen. Reproduction in Domestic Animals. Abstract 45:18

29 Wahjuningsih S. \& Rachmawati A. 2012. The Effect of a-tocopherol on plasma membrane integrity of goat spermatozoa. Journal of Basic and Applied Science Research. 2: 8857-8860. 